\title{
The line between COVID-19 pandemic and rare bone diseases
}

\author{
Luca Sangiorgi $^{1,2}$ (D) Evelise Brizola ${ }^{1}$ (D) - on behalf of the COVID-19 Helpline for Rare Bone Diseases Group
}

Received: 23 September 2020 / Accepted: 15 October 2020 / Published online: 2 November 2020

(C) The Author(s) 2020

The recent outbreak of COVID-19 pandemic had a dramatic global impact for healthcare systems and required a rapid rearrangement of the priorities. In Europe, a disease is defined as rare when it affects no more than 5 in 10,000 people [1]. Between 6000 to 8000 distinct rare diseases exist today affecting around 6-8\% of the population-over 30 million people in Europe are directly involved [2], which number is close to the number of people currently affected with COVID-19 globally (more than 21 million) [3]. Among European countries, Italy has been particularly overwhelmed by COVID-19, with approximately 254,000 infected people and more than 35,000 deaths (WHO report of August 16th 2020) [3]. Suddenly, healthcare workers have found themselves in the front line of this historical moment of emergency [4], and at the same time, their patients started to feel helpless and abandoned, facing delays in medical visits or pre-scheduled surgeries and struggling in refilling even simple pharmacological prescriptions. Patients with rare bone diseases (RBD) have been in need of special care in this emergency situation, starting from seeking access to care and high-quality information. In fact, patients with RBD need careful attentions from a multidisciplinary team since complications can affect different organs and systems (i.e. the musculoskeletal, the pulmonary, the cardiovascular systems).

The priority for experts of RBD has become finding a way to communicate directly with patients and healthcare professionals (who often did not have any previous experience with patients with rare diseases). Thus, the European Reference Network on Rare Bone Diseases (ERN BOND) coordination team and some Italian RBD healthcare professionals have set up a direct and dedicated 24/7 telephone line the "COVID-19

Luca Sangiorgi

luca.sangiorgi@ior.it

1 Department of Rare Skeletal Disorders, IRCCS Istituto Ortopedico Rizzoli, Via Pupilli 1, 40136 Bologna, Italy

2 CLIBI Laboratory, IRCCS Istituto Ortopedico Rizzoli, Bologna, Italy
Helpline for Rare Bone Diseases" [5]. The purpose is to provide experience and knowledge about RBD to patients and healthcare professionals working in the intensive care units and/or COVID-19-devoted wards who are treating or will treat patients affected by RBD, initially focusing on patients with osteogenesis imperfecta. For all patients with RBD, it is crucial to know that they can constantly rely on their primary care physicians and keep these professionals informed about their disease, in particular for those patients who walk with difficulty or cannot even walk independently and already have many other health complications related to their disease.

ERN BOND includes 38 centres from 10 European countries, and the network is actively sharing information to disseminate the best practices for COVID-19 cases and discuss the possibility to make the Helpline initiative available in other participating European countries as well. Considering that Spain, Germany, France and other European countries have also been hit by COVID-19, it is imperative to keep a highly collaborative profile among all countries in order to control the pandemic crisis. For this reason, many helpful initiatives have been implemented both in Italy and across Europe. Further information can be found on the ERN BOND website: http://ernbond.eu/

To date, more than 300 telephone calls and messages have been handled by the COVID-19 Helpline for RBD, and information about cardiopulmonary conditions, orthopedic problems, pharmacological interactions and treatments and other issues were carried out both to healthcare professionals and patients. In some cases, we have followed up patients by remote checking daily for negative evolutions and supporting general practitioners in the decision for referring (or not) patients to the COVID-19 wards.

We have also been frequently contacted by patients affected by rare diseases other than RBD; this clearly demonstrates that dedicated Helplines should be considered valuable and simple tools for reaching out who is in need of specific expertise in these extraordinary situations.

We would like to highlight that in this exact moment, there are more than 761,000 deaths caused by COVID-19 and more than 21,294,000 positive confirmed cases worldwide [3], and 
according to the general population data, approximately $6-8 \%$ of them might be affected by rare diseases. Our successful experience underlines the importance of organizing rapidly concrete initiatives aimed to provide information and expertise remotely to patients and healthcare professionals.

Acknowledgements The COVID-19 Helpline for Rare Bone Diseases Group:

Andrea Vianello, Respiratory Pathophysiology Division, University of Padua, Padua, Italy; Anna Zambrano, Department of Pediatrics, Center for Congenital Osteodystrophy - Sapienza University, Rome, Italy; Annalisa Scopinaro, Italian Federation of Rare Diseases Patients Associations (UNIAMO FIMR); Davide Gatti, Rheumatology Unit, University of Verona, Verona, Italy; Elena Pianigiani, Department of Rare Skeletal Disorders, IRCCS Istituto Ortopedico Rizzoli, Bologna, Italy; Elio Castagnola, Infectious Disease Department, Giannina Gaslini Institute, IRCCS, Genova, Italy; Giampiero I. Baroncelli, Division of Pediatrics, Department of Obstetrics, Gynecology and Pediatrics, University-Hospital, Pisa, Italy; Giovanna Mantovani, Endocrinology Unit, Fondazione IRCCS Ca' Granda Ospedale Maggiore Policlinico, Milan and Department of Clinical Sciences and Community Health, University of Milan, Milano, Italy; Giovanni Adami, Rheumatology Unit, University of Verona, Verona, Italy; Giuseppe Zampino, Rare Diseases and Birth Defects Unit, Dipartimento di Scienza della Salute della Donna, del Bambino e di Sanità Pubblica, Fondazione Policlinico Universitario A. Gemelli IRCCS, Università Cattolica del Sacro Cuore, Rome, Italy; Laura Ruzzini, Department of Paediatric Orthopaedics, Bambino Gesù Children's Hospital, IRCCS, Rome, Italy; Laura Trespidi, Obstetrics and Gynecologic Unit, Fondazione IRCCS Ca' Granda, Ospedale Maggiore Policlinico, Milano, Italy; Leonardo Panzeri, Italian Osteogenesis Imperfecta Association (AS.IT.OI), Olgiate Molgora (LC), Italy; Lorena Casareto, Department of Rare Skeletal Disorders, IRCCS Istituto Ortopedico Rizzoli, Bologna, Italy; Luigi A. Nasto, Department of Paediatric Orthopaedics, IRCCS Giannina Gaslini Institute, Children's Hospital, Genova, Italy; Marco Carbone, Institute for Maternal and Child Health-IRCCS "Burlo Garofolo", University of Trieste, Trieste, Italy; Maria Beatrice Michelis, Department of Paediatric Orthopaedics, IRCCS Giannina Gaslini Institute, Children's Hospital, Genova, Italy; Maria Francesca Bedeschi, Medical Genetic Unit, Fondazione IRCCS Ca' Granda, Ospedale Maggiore Policlinico, Milano, Italy; Maria Luisa Brandi, Department of Experimental Biochemical and Clinical Sciences, University of Florence and Fondazione FIRMO, Florence, Italy; Mauro Celli, Department of Pediatrics, Center for Congenital Osteodystrophy - Sapienza University, Rome, Italy; Michaela V. Gonfiantini, Rare Diseases and Medical Genetics Unit, Bambino Gesù Children's Hospital, IRCCS, Rome, Italy; Paolo Fraschini, Scientific Institute, IRCCS E. Medea, Bosisio Parini (LC), Italy; Sandro Giannini, Department of Medicine, Clinica Medica 1, University of Padova and Regional Centre for Osteoporosis, Padua, Italy; Silvio Boero, Department of Paediatric Orthopaedics, IRCCS Giannina Gaslini Institute, Children's Hospital, Genova, Italy; Stefania Sella, Department of Medicine, Clinica Medica 1, University of Padova, Padua, Italy; Vittorio Landoni, Valduce Hospital - Villa Beretta Rehabilitation Centre, Lecco, Italy.

Authors' contributions LS and EB drafted the manuscript; all authors revised critically and approved the content of the final manuscript.

Funding This publication has received funding from the European Union's Horizon 2020 research and innovation program under grant agreement No 847078 . The materials presented and views expressed here are the responsibility of the authors(s) only. The EC Commissions takes no responsibility for any use made of the information set out.

Data availability Not applicable.

\section{Compliance with ethical standards}

Competing interests The authors declare that they have no competing interests.

Ethics approval and consent to participate Not applicable.

Consent for publication Not applicable.

Code availability Not applicable.

Open Access This article is licensed under a Creative Commons Attribution 4.0 International License, which permits use, sharing, adaptation, distribution and reproduction in any medium or format, as long as you give appropriate credit to the original author(s) and the source, provide a link to the Creative Commons licence, and indicate if changes were made. The images or other third party material in this article are included in the article's Creative Commons licence, unless indicated otherwise in a credit line to the material. If material is not included in the article's Creative Commons licence and your intended use is not permitted by statutory regulation or exceeds the permitted use, you will need to obtain permission directly from the copyright holder. To view a copy of this licence, visit http://creativecommons.org/licenses/by/4.0/.

\section{References}

1. Wakap SN, Lambert DM, Olry A et al (2020) Estimating cumulative point prevalence of rare diseases: analysis of the Orphanet database. Eur J Hum Genet 28:165-173. https://doi.org/10.1038/s41431-019-0508-0

2. Council of the European Union (2009) Council recommendation on action in the field of rare diseases - 2947th employment, social policy, health and consumer affairs - council meeting. https:// eurlex.europa.eu/LexUriServ/LexUriServ.do?uri=OJ:C:2009:151: 0007:0010:EN:PDF. Accessed April 72020

3. World Health Organization, WHO (2020) Coronavirus disease (COVID-2019) situation reports: Situation report - 209. https:// www.who.int/docs/default-source/coronaviruse/situation-reports/ 20200816-covid-19-sitrep-209.pdf?sfvrsn=5dde1ca2_2. Accessed August 312020

4. World Health Organization, WHO (2020) Coronavirus disease (COVID-19) technical guidance: infection prevention and control. https://www.who.int/emergencies/diseases/novel-coronavirus-2019/ technical-guidance/infection-prevention-and-control. Accessed April 72020

5. Brizola E, Adami G, Baroncelli GI et al (2020) Providing highquality care remotely to patients with rare bone diseases during COVID-19 pandemic. Orphanet J Rare Dis 15(1):228. https://doi. org/10.1186/s13023-020-01513-6

Publisher's note Springer Nature remains neutral with regard to jurisdictional claims in published maps and institutional affiliations. 